\title{
Induction in Orthodox Involution Categories (Orthodox Categories, 3) (*) (**).
}

\author{
Marco Grandois (Genova)
}

\begin{abstract}
Sunto. - Si definiscono $i$ morfismi indotti in una categoria involutiva regolare, fattorizzante (dotata di fattorizzazioni epi-mono "uniche") e ortodossa (composizione di endomorfismi idempotenti è idempotente); si prova.ehe in tale ambito l'ortodossia è condizione necessaria e sufficiente per la componibilità degli isomorfismi canonici (indotti dai morfismi identità). Questo lavoro e gli altri della serie saranno utilizzati per studiare le relazioni indotte tra subquozienti in una categoria esatia.
\end{abstract}

\section{Introduction.}

This paper is No. 3 in a series of four $\left({ }^{1}\right)$; in No. 1 the canonical preorder $(\boldsymbol{C})$ and canonical congruence $(\boldsymbol{\Phi})$ for an orthodox category (1.24) are defined (1.26-27); in No. 2 every category Je provided with a regular involution is supplied with an orthodox involution category $\mathcal{H}^{\square}(2.20)$ which we call the orthodox expansion of $\mathcal{H}$.

Here we define induction (3.8) and canonical isomorphisms between subobjects in a factorizing (i.e. having "unique" epic-monic factorizations: see 3.1) orthodox involution category: canonical isomorphisms are composable, and orthodoxy is a necessary and sufficient condition (3.17) for such a fact.

These considerations will be applied in a following paper out of this series, to the canonical symmetrization $\mathscr{H}$ of an exact category $\mathcal{E}$, to supply a "good" definition of induced relations and canonical isomorphisms between subquotients of $\&$ (note that, 3 being a factorizing regular involution category, $\mathfrak{H}^{-}$is still factorizing by 3.7 ).

The hypothesis "Je factorizing" can be erased in 3.5-6 and in the whole section II (but for the last remark in 3.13 ).

Conventions and terminology of Nos. 1, 2 are always effective.

(*) Lavoro compiuto nell'ambito dei gruppi di ricerca del C.N.R.

(**) Entrata in Redazione il 15 Novembre 1976.

$\left({ }^{1}\right)$ The previous papers being: Canonical preorder and congruence in orthodox semigroups and categories (Orthodox Oategories, 1), Boll. Un. Mat. Ital., 13-B (1976), pp. 634-650; Regular and orthodox involution categories (Orthodox Categories, 2), Boll. Un. Mat. Ital., 14-A (1977), pp. 38-48. Item $1 \cdot p$ (resp. $2 \cdot p, 3 \cdot p$ ) is to be found in No. 1 (resp. No. 2, here). 


\section{I. - Monomorphisms and projections.}

3.1. From now on, the regular involution category $\mathcal{H}$ is supposed to be factorizing, that is any morphism $\alpha$ has an epic-monic factorization

$$
\alpha=\mu \circ \pi \quad(\pi \text { epic; } \mu \text { monic })
$$

unique up to isomorphism, the canonical factorization of $\alpha$. Actually, it is easy to see that the uniqueness of these factorizations is here a consequence of the presence of a regular involution, by which any monic (resp. epic) $\alpha$ has a left (resp. right) inverse: $\tilde{\alpha}$. Moreover:

$$
c(\alpha)=\tilde{\alpha} \alpha=\tilde{\pi} \pi=e(\pi), \quad i(\alpha)=\alpha \tilde{\alpha}=\mu \tilde{\mu}=i(\mu) .
$$

3.2. The usual preorder and equivalence relation among monics are written $<_{M}$ and $\sim_{M}\left({ }^{2}\right)$; in each class of equivalent monomorphisms, forcely having all the same codomain $A$, one is chosen to be called a subobject of $A$; in the class of isomorphisms having codomain $A$ we choose always $1_{A}$. Dually are treated the epimorphisms, their preorder and equivalence being written $\prec_{\boldsymbol{P}}$ and $\sim_{\boldsymbol{P}}$; the choice of quotients is here determined by the precedent one, as we want that:

$$
\mu \text { is a subobject of } A \text { iff } \tilde{\mu} \text { is a quotient of } A \text {. }
$$

By the previous assumption, $T e$ is provided with coimages and images: if 3.1 .1 is a canonical factorization of $\alpha \in \mathscr{H}(A, B)$, coim $\alpha$ and $i m \alpha$ are respectively the quotient of $A$ and the subobject of $B$ such that:

$$
\operatorname{coim} \alpha \sim_{P} \pi, \quad i m \alpha \sim_{M} \mu .
$$

By (1), it is easy to see that:

$$
\operatorname{coim} \tilde{\alpha}=(i m \alpha)^{\sim} \text {. }
$$

3.3. Lemma. - Let $\sigma \in \mathfrak{H}(A)=\mathfrak{H}(A, A)$ :

a) $\sigma$ is idempotent iff there exist $\mu, v$ monomorphisms of $H$ such that:

$$
\sigma=\nu \tilde{\mu}, \quad \tilde{\mu} \nu=1
$$

(2) $\mu<_{M} \nu$ iff $\mu$ and $\nu$ are monics in $\mathscr{H}$, and there exists $\nu^{\prime}$ in $\mathscr{H}$ such that $\mu=v v^{\prime}$; $\mu \sim_{M} v$ iff $\mu<_{M^{v}}$ and $v<_{M} \mu$. 
and then

(2)

$$
\tilde{\mu} \sim_{P} \operatorname{coim} \sigma, \quad \nu \sim_{M} i m \sigma
$$

b) $\sigma$ is a projection iff, moreover, $\mu=\nu$.

Proof. - a) From condition (1): $\sigma \sigma=v \tilde{\mu} v \tilde{\mu}=v(\tilde{\mu} v) \tilde{\mu}=v \tilde{\mu}=\sigma$. Vice versa let $\sigma=\nu \tilde{\mu}$ be a canonical factorization of $\sigma$, an idempotent; then

$$
\tilde{\mu} \nu=(\tilde{\nu} \nu) \tilde{\mu} \nu(\tilde{\mu} \mu)=\tilde{v}(\nu \tilde{\mu})(\nu \tilde{\mu}) \mu=\tilde{v}(\nu \tilde{\mu}) \mu=1
$$

b) Sufficiency of condition $\mu=\nu$ being obvious, let us prove its necessity for $\sigma=\tilde{\sigma}$

$$
\mu=\mu \tilde{\nu} \nu=\tilde{\sigma} v=\sigma \nu=\nu \tilde{\mu} \nu=\nu 1=\nu .
$$

3.4. For any object $A$ in $\mathscr{H e}$, let $\boldsymbol{M}_{\mathcal{H}}(A)$ and $\boldsymbol{M}_{\mathcal{H}}^{\prime}(A)$ denote the "set" of $\mathcal{H}$-subobjects of $A$ and, respectively, of $\mathcal{H}$-monomorphisms of codomain $A$, provided with their canonical preorder $\prec_{\boldsymbol{M}}$. We write also $\boldsymbol{M}(A)$ and $\boldsymbol{M}^{\prime}(A)$ if no confusion may arise.

As in [1], 3.18, we consider two mappings

$$
\begin{aligned}
& \boldsymbol{M}_{\mathfrak{H}}(A) \rightarrow \mathfrak{H}_{0}(A), \quad \mu \mapsto i(\mu)=\mu \tilde{\mu} \\
& \mathfrak{H}_{0}(A) \rightarrow \boldsymbol{M}_{\mathfrak{H}}(A), \quad \varepsilon \mapsto i m \varepsilon
\end{aligned}
$$

It is easy to prove these mappings to be reciprocal (use 3.3 to prove that $i(i m \varepsilon)=\varepsilon$ ); moreover, by 3.1.2, (2) takes $e(\alpha)$ in $(\operatorname{coim} \alpha)^{\sim}$ and $i(\alpha)$ in $i m \alpha$. The following conditions are easily seen to be equivalent, for $\mu, v \in \boldsymbol{M}^{\prime}(A)$

$$
\begin{aligned}
& \mu<_{M} v \\
& \mu=v \tilde{v} \boldsymbol{l} \\
& \mu \tilde{\mu}=v \tilde{v} \mu \tilde{\mu} \\
& \mu \tilde{\mu}=\mu \tilde{\mu} \nu \tilde{v} \\
& i(\mu)<i(v) \quad \text { in } \mathscr{H}_{0}(A)
\end{aligned}
$$

Consequently:

$$
\mu \sim_{M} \nu \quad \text { iff } \quad i(\mu)=i(v) .
$$

3.5. For $\mu, v \in \mathbf{M}^{\prime}(A)$, we consider the morphism

$$
\tilde{\nu} \mu: \operatorname{Dom} \mu \rightarrow \operatorname{Dom} \nu \text {. }
$$


The conditions $(2),\left(2^{\prime}\right)$ (resp. $\left.(3),\left(3^{\prime}\right)\right)$ are easily seen to be equivalent:

$\tilde{y} \mu$ is monic

$i(\mu)=i(\mu) i(\nu) i(\mu)$

$\tilde{v} \mu$ is an isomorphism

$i(\mu)=i(\mu) i(v) i(\mu) \quad$ and $\quad i(v)=i(v) i(\mu) i(v)$.

In fact (2) means that:

$$
\mathbf{1}=(\tilde{v} \mu)^{\sim}(\tilde{v} \mu)=\tilde{\mu} v \tilde{y} \mu
$$

which is equivalent to

$$
\mu \tilde{\mu}=\mu \tilde{\mu} \nu \tilde{\nu} \mu \tilde{\mu}
$$

3.6. Defrnition. - For $\mu, \nu \in \boldsymbol{M}^{\prime}(A)$ we write

$$
\mu \mathbf{Q}_{M} \nu
$$

iff the equivalent conditions $3.5 .2-2^{\prime}$ are satisfied; and

$$
\mu \boldsymbol{\Phi}_{M} \nu
$$

iff $\mu \mathbf{\sigma}_{M} \nu$ and $\nu \boldsymbol{\sigma}_{M} \mu$, that is iff $3.5 .3-3^{\prime}$ are true.

Dually, for epics $\pi, \pi^{\prime}$ having the same domain, we write

$$
\begin{array}{lll}
\pi \mathbf{C}_{\boldsymbol{P}} \pi^{\prime} & \text { iff } & \tilde{\pi} \mathbf{C}_{\boldsymbol{M}} \tilde{\pi}^{\prime} \\
\pi \boldsymbol{\Phi}_{\boldsymbol{P}} \boldsymbol{\pi}^{\prime} & \text { iff } & \tilde{\pi} \boldsymbol{\Phi}_{\boldsymbol{M}} \tilde{\pi}^{\prime} .
\end{array}
$$

Relations (1), (2) may be non-transitive in $\boldsymbol{M}^{\prime}(A)\left({ }^{3}\right)$; nevertheless, when $\mathcal{H}$ is orthodox, relations $3.5 .2^{\prime}-3^{\prime}$ on projections $i(\mu), i(v)$ become the domination $\mathbf{\alpha}$ and the canonical congruence $\Phi$ of $\mathcal{T}$ (1.26 and 2.13), which proves that $\boldsymbol{\sigma}_{M}$ is a preorder and $\boldsymbol{\Phi}_{M}$ an equivalence relation: it becomes possible to speak of canonically isomorphic subobjects of $x$ (see the next section). Before, let us ensure of the following fact.

3.7. THEOREM. - If Te is a factorizing regular involution category, its orthodox expansion $\mathfrak{T}^{\square}$ is factorizing.

$\left({ }^{3}\right)$ If $\mathfrak{H}$ is the category of relations among left $R$-modules ( $R$ a ring), relations (2) and (3) are proved ([2], p. 53) to be non-transitive in $\boldsymbol{M}_{\mathfrak{J}}^{\prime}(A \oplus A)$, where $A$ is a non-zero left $R$-module. This is the reason to "replace" $\mathcal{H}$ by $\mathcal{H}^{a}$, an orthodox category. 
Proof. - Let

$$
\alpha^{\prime}=(\alpha, S, T):(A, S) \rightarrow(B, T)
$$

be a morphism in $\mathfrak{H}^{\square}$, and

$$
\alpha=\mu \pi, \quad \operatorname{Cod} \pi=\operatorname{Dom} \mu=C
$$

a canonical factorization of $\alpha$ in $\mathscr{K}$; $\tilde{\pi} \pi=\tilde{\alpha} \alpha \in S$, hence (2.19) $\pi_{5}(S)$ is an idempotent involution subsemigroup of $O$ and

$$
\pi^{\prime}=\left(\pi, S, \pi_{\square}^{\top}(S)\right):(A, S) \rightarrow\left(C, \pi_{\square}(S)\right)
$$

is an epimorphism (2.23) in $H^{\square}$; likewise

$$
\mu^{\prime}=\left(\mu, \mu^{\square}(T), T\right):\left(C, \mu^{\square}(T)\right) \rightarrow(B, T)
$$

is a monomorphism, Moreover:

$$
\begin{aligned}
& \pi_{\mathrm{a}}(S)=\pi S \tilde{\pi}=\tilde{\mu}(\mu \pi S \tilde{\pi} \tilde{\mu}) \mu=\mu^{\square}\left(\alpha_{\mathrm{a}}(S)\right) \subset \mu^{\square}(T) \\
& \mu^{\mathrm{\square}}(T)=\tilde{\mu} T \mu=\pi(\tilde{\pi} \tilde{\mu} T \mu \pi) \tilde{\pi}=\pi_{\square}\left(\alpha^{\square}(T)\right) \subset \pi_{\mathrm{a}}(S)
\end{aligned}
$$

which proves that $\pi_{\square}(S)=\mu^{\square}(T)$; thus $\pi^{\prime}$ and $\mu^{\prime}$ are composable, and

$$
\alpha^{\prime}=\mu^{\prime} \pi^{\prime}
$$

the uniqueness of this factorization being a consequence of the regular involution on $\mathfrak{H E}^{\mathrm{a}}(3.1)$

\section{II. - Induction in factorizing orthodox involution categories.}

In this section, te is always a factorizing (3.1) orthodox involution category (2.13), provided with its canonical preorder (or domination) $\mathbf{U}$ and its canonical congruence $\boldsymbol{\Phi}$ (2.13). $A$ is an object in $\mathcal{H e}$.

3.8. Definition. - If in the following (possibly non-commutative) diagram

(1)

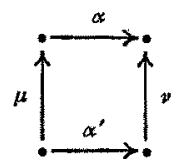


$\mu$ and $\nu$ are monics, we shall say that $\alpha^{\prime}$ is induced by $\alpha$ on $\mu$ and $\nu$ iff:

$$
\alpha^{\prime} \mathbf{C} \tilde{v} \alpha \mu
$$

This definition will be justified in section IV (3.19-21).

3.9. Induction respects identities ( 1 induces 1 on $\mu$ and $\mu$ ), involution ( $\alpha^{r}$ is induced by $\alpha$ on $\mu$ and $\nu$ iff $\tilde{\alpha}^{\prime}$ is induced by $\tilde{\alpha}$ on $\nu$ and $\mu$ ) and composition:

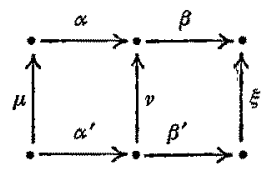

$\alpha^{\prime}$ and $\beta^{\prime}$ being induced by $\alpha$ and $\beta$ on $\mu$ and $\nu, \nu$ and $\xi$, we have

$$
\beta^{\prime} \alpha^{\prime} \underline{\mathbf{I}}(\tilde{\xi} \beta \nu)(\tilde{v} \alpha \mu)=\xi \xi(\nu \tilde{v}) \alpha \mu \mathbf{\mathbf { T }} \xi \beta \alpha \mu
$$

which proves that $\beta^{\prime} \alpha^{\prime}$ is induced by $\beta \alpha$ on $\mu$ and $\xi$.

3.10. If $\alpha$ induces an isomorphism $\alpha^{\prime}$ on $\mu$ and $\nu, \alpha^{\prime} \mathbf{C} \tilde{\nu} \alpha \mu$ and, by $1.27 g$,

$$
\alpha^{t}=\tilde{\nu} \alpha \mu
$$

The induced isomorphism is then unique (when existing), and maximum among induced morphisms.

Composition of induced isomorphisms is still an induced morphism by 3.9 , and obviously an isomorphism, hence the only possible.

3.11. Let us consider, particularly, a fixed object $A$ and its subobjects: $\mu$ and $\nu$ being such, any morphism $\alpha^{\prime}$ induced by $1_{A}$ on $\mu$ and $\nu$ :

$$
\alpha^{\prime} \mathbf{Q} \tilde{\nu} \mu
$$

will be said induced on $\mu$ and $v$.

3.12. The following conditions on $\mu, v$, subobjects of $A$, are equivalent:

(1) there is an isomorphism induced on $\mu$ and $\nu$ (by $1_{A}$ ),

(2) $\tilde{y} \mu$ is an isomorphism,

(3) $\mu \Phi_{M} v$. 
In fact (1) and (2) are equivalent by 3.10 (take $\alpha=1_{A}$ ), (2) and (3) by 3.6.2. These conditions being satisfied, the isomorphism induced on $\mu$ and $v$ is forcely $\tilde{\nu}_{\mu}$ (3.10.1): it will be called the eanonical isomorphism from $\mu$ to $v$ while $\mu$ and $v$ will be said to be canonically isomorphic (as subobjects of $A$ ). Canonical isomorphisms are composable by 3.10 .

Having to use such isomorphisms in the case of a factorizing regular involution category (possibly non-orthodox), they will be called pseudo-canonical isomorphisms, in order to recall the possibility of non-componibility (see 3.19).

3.13. We consider now the inverse category $\mathfrak{H}=\overline{\mathfrak{H}} / \boldsymbol{\Phi}$ : monomorphisms in $\overline{\mathfrak{H}}$ are just the images of monomorphisms in $\mathscr{H}(1.27 h)$, thus $\overline{\mathcal{H}}$ is factorizing; moreover $\alpha$ induces $\alpha^{\prime}$ on $\mu$ and $v$ in $\mathscr{H}$ iff $\bar{\alpha}$ induces $\bar{\alpha}^{\prime}$ on $\bar{\mu}$ and $\bar{v}$ in $\overline{\mathscr{H}}(1.27 e)$. In $\overline{\mathscr{H}}$, as in every factorizing inverse category, one has the advantage that, $\boldsymbol{\alpha}$ being an order, there exists a unique maximum induced morphism (by $\bar{\alpha}$ on $\bar{\mu}$ and $\bar{v}$ : precisely $\tilde{\bar{\nu}} \bar{\alpha} \bar{\mu}$ ); composition of maximum induced morphisms may, of course, not be maximum, but in the case of induced isomorphisms.

Remark also that, for $\bar{\mu}, \bar{v} \in \boldsymbol{M}_{\mathfrak{H}}^{\prime}(A)$ :

$$
\begin{array}{lll}
\bar{\mu} \mathbf{O}_{M} \bar{\nu} & \text { iff } & \bar{\mu} \prec_{M} \bar{\nu} \\
\bar{\mu} \boldsymbol{\Phi}_{M} \bar{\nu} & \text { iff } & \bar{\mu} \sim_{M} \bar{\nu}
\end{array}
$$

namely $\bar{\mu} \mathbf{⿴ 囗 十}_{M} \bar{\nu}$ iff $i(\bar{\mu}) \mathbf{Q} i(\bar{\nu})$ by $3.6 .1 ; \bar{\mu}<_{M} \bar{\nu}$ iff $i(\bar{\mu})<i(\bar{\nu})$ by 3.4; now $\mathbf{\alpha}$ and $<$ coincide in $\overrightarrow{\mathfrak{F}}_{0}(\boldsymbol{A})$, commutative idempotent semigroup.

Last, $\boldsymbol{M}_{\overline{\mathfrak{K}}}(A)$ has intersections, as $\overline{\mathfrak{H}}_{0}(A)$ :

$$
\bar{\mu} \cap_{M} \bar{\nu}=i m(i(\tilde{\mu}) i(\bar{\nu})) .
$$

\section{III. - Necessity of orthodoxy.}

Let $\mathfrak{H}$ be a factorizing regular involution category, $A$ an object to $J$.

3.14. We associate to any set $M$ of subobjects of $A$ the involution subsemigroup $\left(^{4}\right) \boldsymbol{S}(M)$ of $\mathcal{H}(A)$ generated by all the projections $\mu \tilde{\mu}$, for $\mu \in M$; we associate to any involution subsemigroup $S$ of $\mathscr{H}(A)$ the set $\boldsymbol{M}(S)$ of subobjects $\mu$ of $A$ such that $\mu \tilde{\mu} \in S\left({ }^{5}\right)$.

(4) We recall (see 2.19 , footnote $\left(^{4}\right)$ ) that an involution subsemigroup is here a subsemigroup stable for involution and having the same identity of the semigroup in object.

(5) Equivalent condition: $\mu=i m(\alpha)$, for some $\alpha \in S$; in fact, $\mu$ being a subobject we have $\mu=i m(\mu \tilde{\mu})$; viceversa, $\mu=i m(\alpha)$, with $\alpha \in S$, implies $\mu \tilde{\mu}=\alpha \tilde{\alpha} \in S$. 
3.15. These mappings constitute a Galois connection $[3,4]$ :

$$
\begin{array}{ll}
M \subset M^{\prime} \Rightarrow S(M) \subset S\left(M^{\prime}\right), & S \subset S^{\prime} \Rightarrow M(S) \subset M\left(S^{\prime}\right) \\
M S(M) \supset M & S M(S) \subset S .
\end{array}
$$

An involution subsemigroup $S$ of $\mathfrak{K}(A)$ is closed in this connection (i.e. $S=S \boldsymbol{S M}(S)$ ) iff it is generated by its projections; a characterization of closed sets of subobjects of $A$ will be given in No. 4 ,

3.16. Marn Theorem. - Let $S$ be an involution subsemigroup of $\mathscr{H}(A)$, generated by its projections; if $M=\boldsymbol{M}(S)$, the following conditions are equivalent

(1) $S$ is idempotent

(2) pseudo-canonical isomorphisms (3.12) among elements of $M$ (as subobjects of $A)$ are composable; i.e., if $\mu_{i} \in M(i=1,2,3)$ and $\tilde{\mu}_{2} \mu_{1}, \tilde{\mu}_{3} \mu_{2}$ are isomorphisms then $\left(\tilde{\mu}_{3} \mu_{2}\right)\left(\tilde{\mu}_{2} \mu_{1}\right)=\tilde{\mu}_{3} \mu_{1}$.

Proof. $-(1) \Rightarrow(2)$. Let $\mathbb{S}$ be idempotent, $\mu_{i}: L_{i} \rightarrow A$ elements of $M(i=1,2,3)$ and $\tilde{\mu}_{2} \mu_{1}, \tilde{\mu}_{3} \mu_{2}$ isomorphisms.

In $\mathcal{H}^{\mathrm{a}}(2.20)$ we consider the following objects and monomorphisms $(i=1,2,3)$ :

$$
\begin{aligned}
& A^{\prime}=(A, S), \quad L_{i}^{\prime}=\left(L_{i}, \mu_{i}^{\square}(S)\right) \\
& \mu_{i}^{\prime}=\left(\mu_{i}, \mu_{i}^{\square}(S), S\right)
\end{aligned}
$$

(which is justified by 2.23 , as $M=M(S)$ and $\mu_{i} \in M$ gives $\mu_{i} \tilde{\mu}_{i} \in S$ ).

In $\mathfrak{H}^{\square}$, a factorizing orthodox involution category, canonical isomorphisms are composable (3.10) so that

$$
\left(\tilde{\mu}_{3}^{\prime} \mu_{2}^{\prime}\right)\left(\tilde{\mu}_{2}^{\prime} \mu_{1}^{\prime}\right)=\tilde{\mu}_{3}^{\prime} \mu_{1}^{\prime}
$$

and in $\mathfrak{H e}$

$$
\left(\tilde{\mu}_{3} \mu_{2}\right)\left(\tilde{\mu}_{2} \mu_{1}\right)=\tilde{\mu}_{3} \mu_{1}
$$

A direct proof, not using $\mathfrak{H}^{\square}$, will be given in 3.18 .

$(2) \Rightarrow(1)$. Let $\varepsilon$ and $\sigma$ be respectively a projection and an idempotent of $S$; by 2.6 it is sufficient to prove that $\sigma \varepsilon$ is idempotent.

First, $\sigma$ being idempotent in $S$, we have (3.3a) two monomorphisms $\mu, \nu$ of $\mathscr{H}$ such that

$$
\sigma=\nu \tilde{\mu}, \quad \tilde{\mu} v=1
$$


moreover $\mu \tilde{\mu}=\tilde{\sigma} \sigma$ and $\nu \tilde{\nu}=\sigma \tilde{\sigma}$ are in $S$ (that is, $\mu$ and $\nu$-which we cannot pretend to be both subobjects-are equivalent to elements of $M$, which we shall write $\mu$, $v \in M^{\prime}$ ).

In the same way, $(\mu \tilde{\mu}) \varepsilon$, idempotent by 2.3 , has a factorization:

$$
\mu \tilde{\mu} \varepsilon=\nu_{1} \tilde{\mu}_{1}, \quad \tilde{\mu}_{1} \nu_{1}=1 \quad\left(\mu_{1}, \nu_{1} \in M^{\prime}\right)
$$

and we have the following (possibly non-commutative) diagram:

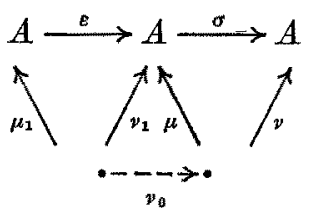

From

$$
\nu_{1} \sim_{M} i m(\mu \tilde{\mu} \varepsilon) \prec_{M} \mu
$$

it follows the existence of a monomorphism $\nu_{0}$ in $\mathscr{H}$ such that $\nu_{1}=\mu v_{0}$. Hence:

$$
\sigma \varepsilon=\nu \tilde{\mu} \varepsilon=\nu \tilde{\mu}(\mu \tilde{\mu} \varepsilon)=\nu \tilde{\mu} v_{1} \tilde{\mu}_{1}=v \tilde{\mu} \mu v_{0} \tilde{\mu}_{1}=\left(\nu v_{0}\right) \tilde{\mu}_{1} .
$$

we prove that $\nu v_{0} \in M^{\prime}$; by (5):

$$
\nu v_{0} \tilde{v}_{0} \tilde{v}=\nu(\tilde{v} \mu) v_{0} \tilde{v}_{0}(\tilde{\mu} v) \tilde{v}=(\nu \tilde{v})\left(v_{1} \tilde{v}_{1}\right)(v \tilde{v})
$$

and the last product is in $S$, as $v, v_{1} \in M^{\prime}$.

We use now the componibility of pseudo-canonical isomorphisms among elements of $M^{\prime}$ (immediately following from the same property for $M$ ), for $\mu_{1}, v_{1},\left(y v_{0}\right)$

$$
\begin{gathered}
\tilde{\nu}_{1} \mu_{1}=1 \quad \text { by }(6) \\
\left(v v_{0}\right) \sim \nu_{1}=\tilde{\nu_{0}} \tilde{y} \mu v_{0}=\tilde{v}_{0} \nu_{0}=1
\end{gathered}
$$

hence $\left(v v_{0}\right)^{\sim} \mu_{1}=1$. Last, $\sigma \varepsilon$ is idempotent by (9) and $3.2 a$.

3.17. CoRollaRY. - If $\mathscr{H}$ is a factorizing regular involution category, its pseudocanonical isomorphisms among subobjects are composable iff He is orthodox.

3.18. Let us give a direct proof of $(1) \Rightarrow(2)$ in 3.16 .

We have $\mu_{1}, \mu_{2}, \mu_{3} \in M, t=\tilde{\mu}_{2} \mu_{1}$ and $\varkappa=\tilde{\mu}_{3} \mu_{2}$ being isomorphisms; we want to 
prove that $\varkappa=\tilde{\mu}_{3} \mu_{1}$. Now the morphism (2)

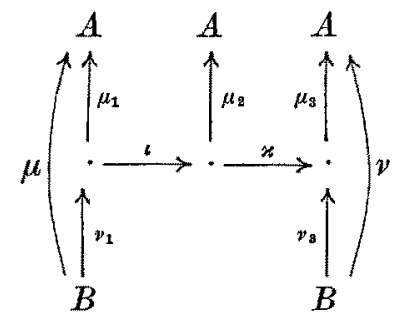

$$
\mu_{3} \varkappa\left(\tilde{\mu}_{1}=\left(\mu_{3} \tilde{\mu}_{3}\right)\left(\mu_{2} \tilde{\mu}_{2}\right)\left(\mu_{1} \tilde{\mu}_{1}\right)\right.
$$

is in $S$, then an idempotent; by $3.3 a$ there exist monics $\mu, v$ such that

$$
\begin{aligned}
& \mu_{3} \varkappa\left(\tilde{\mu}_{1}=v \tilde{\mu}, \quad \tilde{\mu} v=1\right. \\
& \nu \sim i m\left(\mu_{3} \varkappa \iota \tilde{\mu}_{1}\right)<\mu_{3} \\
& \mu \sim i m\left(\mu_{1} \tilde{\imath} \tilde{\mu}_{3}\right)<\mu_{1}
\end{aligned}
$$

and we have monomorphisms $v_{1}, v_{3}$ such that

$$
\mu=\mu_{1} \nu_{1}, \quad v=\mu_{3} v_{3}
$$

Hence:

$$
\varkappa=\tilde{\mu}_{3}\left(\mu_{3} \varkappa\left(\tilde{\mu}_{1}\right) \mu_{1}=\tilde{\mu}_{3} v \tilde{\mu} \mu_{1}=\tilde{\mu}_{3} \mu_{3} v_{3} \tilde{v}_{1} \tilde{\mu}_{1} \mu_{1}=v_{3} \tilde{v}_{1}\right.
$$

which proves that $v_{1}, v_{3}$ are isomorphisms so that:

$$
\tilde{\mu}_{3} \mu_{1}=\nu\left(\tilde{\nu}_{3} \tilde{\mu}_{3} \mu_{1} \nu_{1}\right) \tilde{\nu}_{1}=\nu_{3}(\tilde{\nu} \mu) \tilde{\nu}_{1}=\nu_{3} \tilde{\nu}_{1}=\varkappa \iota .
$$

\section{IV. - Why to define induction by domination.}

3.19. Let Te be a (factorizing) orthodox involution category: the necessity of orthodoxy has been proved in the preceding section, just in order to preserve the componibility of pesudo-canonical isomorphisms, whose definition does not require domination. We want now to justify our definition of induction (3.8).

3.20. We assume that a notion of non-univocal induction $\left(^{6}\right)$ has been defined (which we call *-induction to avoid confusion with 3.8 ) so that:

(6) This means that, for any triple $(\alpha, \mu, v)$ where $\alpha$ is a morphism, $\mu$ and $v$ are monics and $\operatorname{Cod} \mu=\operatorname{Dom} \alpha, \operatorname{Cod} \nu=\operatorname{Cod} \alpha$, a set $I(\alpha, \mu, v) \subset \operatorname{Te}(\operatorname{Dom} \mu, \operatorname{Dom} \nu)$ is assigned, whose elements we say induced by $\alpha$ on $\mu$ and $\nu$. 
a) in any diagram 3.8.1 $\tilde{\nu} \alpha \mu$ is $*$-induced by $\alpha$ on $\mu$ and $\nu$;

b) composition is respected (i.e. in any diagram 3.9.1, if $\alpha^{\prime}$ and $\beta^{\prime}$ are respectively $*$-induced by $\alpha$ on $\mu$ and $\nu$, and by $\beta$ on $\nu$ and $\xi$, then $\beta^{\prime} \alpha^{\prime}$ is $*$-induced by $\beta \alpha$ on $\mu$ and $\xi)$.

We want to prove that any $\alpha^{\prime} \mathbf{C} \tilde{\nu} \alpha \mu$ is $*$-induced by $\alpha$ on $\mu$ and $\nu$.

In other words, our definition of induction (3.8) will result the " more restricted " one among those which satisfy $a$ ) and $b$ ).

3.21. Let $\alpha^{\prime} \mathbf{Q} \tilde{\nu} \alpha \mu(3.8 .1)$; there exist idempotents $\sigma, \tau$ such that

$$
\alpha^{\prime}=\tau \tilde{\nu} \alpha \mu \sigma=\tilde{\nu}(\nu \tau \tilde{\nu}) \alpha(\mu \sigma \tilde{\mu}) \mu .
$$

Now $\mu \sigma \tilde{\mu}=\mu_{\mathrm{a}}(\sigma)$ and $\nu \tau \tilde{v}=\nu_{\mathrm{a}}(\tau)$ are idempotent (2.18) hence (2.4) composition of two projections, so that $(3.3 b)$ we can write:

$$
\mu \sigma \tilde{\mu}=\left(\xi_{2} \xi_{2}\right)\left(\xi_{1} \xi_{1}\right), \quad \nu \tau \tilde{\nu}=\left(\xi_{4} \xi_{4}\right)\left(\xi_{3} \xi_{3}\right)
$$

where all $\xi_{i}$ are monies; in the diagram

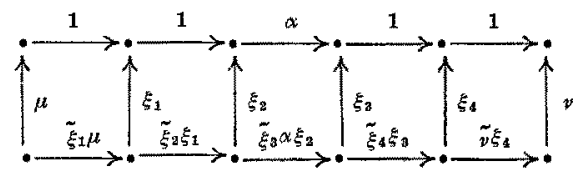

$\tilde{\xi}_{1} \mu$ is *-induced by 1 on $\mu$ and $\xi_{1}(3.20 a)$, and so on; by $3.20 b$, the composition of the low row in (3) is *-induced by $\alpha$ on $\mu$ and $\nu$; by (1) and (2) this compositon is just $\alpha^{\prime}$.

\section{V. - Characterizations of domination in orthodox involution categories.}

$\mathcal{H}$ is an orthodox involution category; $A$ and $B$ are objects in $\mathcal{H}$.

3.22. LemMa. - If $\mu, \alpha \in \mathcal{H}(A, B)$ and $\mu$ is monic, the following conditions are equivalent:

a) $\mu \mathbf{O} \alpha$;

b) $\mu \Phi \propto$

c) $\tilde{\alpha} \mu=1_{A}\left(\tilde{\mu} \alpha=1_{A}\right)$.

If they are satisfied $\alpha$ too is monic, and $\mu \Phi_{M} \alpha$; if $\mathscr{H}$ is inverse, the preceding conditions are equivalent to: $\mu=\alpha$. 
Proof. $-a) \Rightarrow c): 1=\tilde{\mu} \mu(\mathbf{\alpha} \tilde{\alpha}$, hence $1=\tilde{\alpha} \mu . \quad c) \Rightarrow b): \tilde{\alpha} \mu=1$ gives $\mu=\mu \tilde{\alpha} \mu$ and $\alpha=\alpha \tilde{\mu} \alpha . \quad b\rangle \Rightarrow a)$ and the last remark are obvious $(1.27 g)$.

3.23. Lemma. - Let $\mu, v \in M_{\mathfrak{H C}}^{\prime}(A)$; the following are equivalent:

a) $\mu \mathbf{G}_{M} v$;

b) there exists a morphism $\alpha$ such that $\mu \mathbf{\Phi} \nu \alpha$;

c) there exists a morphism $\beta$ such that $\mu \boldsymbol{\Phi} \nu \beta$.

In such a case $\alpha \mathbf{\Phi} \beta \mathbf{\Phi} \tilde{v} \mu$ are monics. Moreover, $\mu \mathbf{\Phi}_{M} \nu$ iff there exists an isomorphism $\iota$ such that $\mu \Phi v$, and in such a case $\iota=\tilde{v} \mu$.

Proof. $-a) \Rightarrow b$ ): take $\alpha=\tilde{\nu} \mu: \mu=\mu \tilde{\mu} \mu \mathbf{\mathbf { C }} v \tilde{\nu} \mu=v \alpha . \quad b) \Rightarrow c)$ : if $\mu \mathbf{\mathbf { C }} v \alpha$ then $\mu \boldsymbol{\Phi} \nu \propto$ by 3.22 ; moreover $\alpha \boldsymbol{\Phi} \tilde{\nu} \mu$, which is monic by 3.6. $c) \Rightarrow a): \mu \tilde{\mu} \boldsymbol{\Phi} v \beta \tilde{\beta} \tilde{\nu} \mathbf{\Phi} v \tilde{\nu}$. The last assertion can be seen in a similar way; for the uniqueness of $\iota$, recall that Ф-equivalent isomorphisms coincide $(1.27 \mathrm{~g})$.

3.24. Proposttion. - Let $\alpha, \alpha^{\prime} \in \mathfrak{H}(A, B)$ have canonical factorizations:

$$
\alpha=\mu \pi, \quad \alpha^{\prime}=\mu^{\prime} \pi^{\prime}
$$

The following conditions are equivalent:

a) $\alpha \mathbf{G} \alpha^{\prime}\left(\operatorname{resp} . \alpha \boldsymbol{\Phi} \alpha^{\prime}\right)$

b) $\pi \mathbf{\alpha}_{P} \pi^{\prime}, \mu \mathbf{C}_{M} \mu^{\prime}$ and $\pi^{\prime} \tilde{\pi} \boldsymbol{\Phi} \tilde{\mu}^{\prime} \mu$ (resp. $\pi \boldsymbol{\Phi}_{\boldsymbol{P}} \pi^{\prime}, \mu \boldsymbol{\Phi}_{M} \mu^{\prime}, \pi^{\prime} \tilde{\pi}=\tilde{\mu}^{\prime} \mu$ );

c) $\pi^{\prime} \tilde{\pi}, \tilde{\mu}^{\prime} \mu$ are $\boldsymbol{\Phi}$-equivalent monics (resp. coincident isomorphisms);

d) there is some monic (resp. some isomorphism) $v: \operatorname{Cod} \pi \rightarrow \operatorname{Cod} \pi^{\prime}$ such that: $v \pi \Phi \pi^{\prime}$ and $\mu^{\prime} \nu \Phi \mu$.

If these conditions are satisfied:

$$
\left.{ }_{\nu} \boldsymbol{\Phi} \pi^{\prime} \tilde{\pi} \boldsymbol{\Phi} \tilde{\mu}^{\prime} \mu \quad \text { (resp. } v=\pi^{\prime} \tilde{\pi}=\tilde{\mu}^{\prime} \mu\right)
$$

Proof. $-a) \Rightarrow b)$ : it is obvious that $\pi \mathbf{U}_{P} \pi^{\prime}$ and $\mu \mathbf{U}_{M} \mu^{\prime}$; moreover:

$$
\begin{array}{ll}
\pi^{\prime} \tilde{\pi}=\pi^{\prime} \tilde{\pi} \tilde{\mu} \mu & \mathbf{Q} \pi^{\prime} \tilde{\pi}^{\prime} \tilde{\mu}^{\prime} \mu=\tilde{\mu}^{\prime} \mu \\
\pi^{\prime} \tilde{\pi}=\tilde{\mu}^{\prime} \mu^{\prime} \pi^{\prime} \tilde{\pi} & \mathbf{D} \tilde{\mu}^{\prime} \mu \delta \tilde{\pi}=\tilde{\mu}^{\prime} \mu .
\end{array}
$$

$b) \Leftrightarrow c)$ : obvious by definition of $\left.\mathbf{a}_{M}, \mathbf{a}_{P}(3.6) . b\right) \Rightarrow d$ ) and the last remark (3) follow from 3.23. $d) \Rightarrow a): \alpha=\mu \pi \Phi \mu^{\prime} v \pi \Phi \mu^{\prime} \pi^{\prime}=\alpha^{\prime}$. The conditions in parenthesis can be treated analogously; as in 3.23 , apply $1.27 \mathrm{~g}$. 


\section{REFERENCES}

[1] M. Grandis, Symétrisations de catégories et factorisations quaternaires, Atti Accad. Naz. Lincei, Mem. Cl. Sci. Fis. Mat. Natur., 14 (sez. 1) (1977), pp. 133-207.

[2] S. MaC LANE, Homology, Springer, 1963.

[3] S. MaC LANE, Categories for the working mathematician, Springer, 1971.

[4] O. ORE: Galois connexions, Transact. of the A.M.S., 55 (1944), pp. 493-513. 